\title{
Prevalência de Violência Física Relatada contra Crianças em uma População de Ambulatório Pediátrico ${ }^{1}$
}

\section{Prevalence of Reported Child Abuse by Parents in a Pediatric Out-Patient Population}

\author{
Adriana Davoli;; Fortunato Antonio B. Palhares²; Heleno R. Corrêa-Filho2; \\ Ana Laura V. Dias²; Adriana B. Antunes²; Julian F. Serpa² \& Patrícia Schincariof
}

DAVOLI, A.; PALHARES, F. A. B.; CORREA-FILHO, H. R.; DIAS, A. L. V.; ANTUNES, A. B.; SERPA,J. F. \& SCHINCARIOL, P. Prevalence of Reported Child Abuse by Parents in a Pediatric Out-Patient Population. Cad. Saúde Públ., Rio de Janeiro, 10(1): 92-98, Jan/Mar, 1994.

A cross-sectional study was carried out to estimate prevalence of child abuse with battering among patients under age 14 for alleged disciplinary purposes. The reference population were children attending pediatric out-patient services at the University Hospital in Campinas, State of São Paulo, Brazil, a city of one million inhabitants with universal coverage in terms of accessing health care in the unit under study. Interviews were carried out with 130 parents or guardians in a randomly selected group according to order of attendance, and the prevalence of child abuse was estimated at $10.8 \%$, considering as positive cases those where parents referred child battering under age one year or where there was evidence of bodily lesion over age one year. $A$ validation study for the experimental questionnaire is proposed in order to allow for future estimation of population prevalence or incidence. The use of qualitative methods is also recommended as a means of gathering detailed data on socio-psychological determination for the supposedly disciplinary aggression with child abuse.

key words: Cross-Sectional Studies; Data Collection; Survey Methods; Child; Child Abuse; Mother-Child Relations; Parent-Child Relations

\section{INTRODUÇÃO}

A idéia de que a criança é um ser imperfeito, rebelde e intransigente está presente há séculos nas civilizações. Aristóteles (Charlot, 1986) dizia: "a criança é, por natureza, destinada a obedecer ao adulto". Segundo Rosseau (Charlot, 1986), "a infância é, por excelência, o período em que germinam o erro e o vício". Para Kant (Charlot, 1986), "as crianças estão, por natureza, em estado de incapacidade, e os

\footnotetext{
${ }^{1}$ Este trabalho foi desenvolvido com a verba do Fundo de Apoio ao Ensino e Pesquisa da Universidade Estadual de Campinas (193/90, 235/91).

${ }^{2}$ Departamento de Medicina Legal da Faculdade de Ciências Médicas da Universidade Estadual de Campinas. Cidade Universitária Zeferino Vaz. Caixa Postal 6111. Campinas, SP, 13083-970, Brasil.
}

pais são seus tutores naturais". A partir desta visão foi se delineando o lugar da criança na sociedade moderna.

A autoridade dos adultos sobre a criança tem na família sua representação mais evidente. Aos pais é conferido o dever de educação e proteção, e o direito de responder pelos filhos até que estes alcancem a maioridade legal.

A família é considerada um local sagrado de formação e agregação de seus membros. A inviolabilidade do espaço familiar esconde situações de violência, dificultando a intervenção para se garantir os direitos de seus membros.

$\mathrm{Na}$ interação do maior de idade com o menor de idade, muitas vezes ocorre a exacerbação da autoridade do primeiro sobre o segundo, levando à situação de abuso. O abuso contra a criança ocorre de diversas maneiras: negligência nos 
cuidados e proteção; abandono; agressão física, sexual ou emocional.

Este fenômeno, apesar de ter na família o seu local privilegiado de ocorrência, é pouco estudado no Brasil (Baeza-Herrera et al., 1986).

A violência contra a criança se insere nos âmbitos ético e legal, devido ao comprometimento da dignidade e do respeito à criança. Giovannoni (1974) coloca: "maus-tratos constituem uma exorbitância no sentido de controlar, disciplinar e punir seu filho, enquanto a negligência representa o fracasso no desempenho de suas funções paternas, inclusive a supervisão, alimentação e proteção".

A agressão física contra a criança é, dentre os vários tipos de violência, uma das que mais nos chama a atenção, pela gravidade de suas conseqüências, bem como por sua tácita aceitação e impunidade. Schmitt \& Kempe (1975) comentaram que crianças agredidas não-tratadas freqüentemente tornam-se delinqüentes, assassinos e agressores da próxima geração de crianças.

Kempe et al. (1962) introduziram a expressão "síndrome da criança espancada" para denominar um conjunto de sinais encontrados em crianças agredidas: hematoma subdural, fraturas múltiplas e sem explicações em diferentes estágios de consolidação, falhas no desenvolvimento, edema e/ou hematoma dos tecidos moles. Na ocorrência de violências contra a criança não se encontrará, necessariamente, a multiplicidade de lesões como as descritas por Kempe (Azevedo \& Guerra, 1989).

Garbarino \& Gilliam (1981) definem a violência contra a criança como o "dano físico não-acidental que é o resultado de atos (ou omissões) de pais ou responsável(is), que violam os padrões da comunidade no que diz respeito aos cuidados com a criança”.

Gil (1986) considera todo e qualquer emprego de força física intencional como abusivo.

$\mathrm{O}$ fator intencionalidade, presente na definição dos autores citados, é de difícil verificação, visto que comportamentos que, num primeiro momento, possam parecer acidentais, podem ter sido determinados por elementos intencionais inconscientes por parte do abusador.

O conceito de violência, pela definição de Garbarino \& Gilliam (1981), é relativizado pela valorização dos aspectos culturais. Portanto, cabe à ciência, juntamente com a cultura, a definição de violência contra a criança. "Falando-se de uma forma mais explícita, este conhecimento científico deve ser acrescido ao critério estabelecido pelas convicções acerca do cuidado com as crianças baseado nos costumes" (Azevedo \& Guerra, 1989).

No Brasil, a utilização da força física como medida disciplinar é parte integrante dos costumes pedagógicos, transmitidos nas famílias de geração a geração. O limite entre disciplina e violência é definido pelos padrões comunitários de tolerância ao uso da força física contra crianças.

\section{OBJETIVOS}

Este trabalho visa investigar a prevalência da utilização da violência física enquanto medida disciplinar numa dada população. Acrescenta-se a este atestar a hipótese de que, no sentido de disciplinar e educar, a força física é utilizada quando outros métodos disciplinares (não-físicos) mostram-se ineficientes, como conversar ou chamar a atenção.

Pretende-se descrever as características sóciodemográficas, a relação dos entrevistados com seus pais e cônjuge e o uso da força física em crianças consideradas diferentes.

\section{METODOLOGIA}

Considerando-se a dificuldade anteriormente mencionada de se avaliar a violência pelo critério de intencionalidade, bem como a nãoabrangência do conceito de Gil (1986) para a realidade brasileira, a partir do exposto por Garbarino \& Gilliam (1981) elaboramos um conceito operacional de violência para este projeto. Tal conceito tem por base a materialidade de atos contra a criança, como marcas e lesões relatadas pelos entrevistados, que violam os padrões comunitários do uso de força física em crianças, bem como considerações científicas sobre o desenvolvimento infantil. Foi definido que a utilização de força física contra crianças menores de 1 ano seria violência, uma vez que só a partir desta idade a criança se relaciona com o mundo externo, necessitando, 
portanto, de disciplina. A este respeito, Tulha (1979) diz que: "depois que a criança completa o primeiro ano de vida, ela já tem uma noção de si mesma como indivíduo personalizado e já descobriu muitos aspectos da realidade com a qual ela joga (...) Com isso, a mãe, que antes se dedicava quase inteiramente a satisfazer a criança, agora passa a limitá-la".

Considerando o exposto, a agressão física, enquanto violência, será vista pelos autores como a existência de um relato positivo por parte do responsável, em informações obtidas através de questionário, independente de verificação clínica ou legal de:

- qualquer uso de força física contra a criança que cause lesão. A lesão será considerada como todo sinal de dano físico causado à criança, de reconhecimento por leigos e visível a olho nu, como hematomas, ferimentos, marcas;

- emprego de força física contra menores de 1 ano de idade, independente de ocorrência de lesão física.

A amostra da pesquisa consistiu de 130 entrevistas, calculadas com base em dados retrospectivos sobre o total de consultas mensais executadas no Ambulatório Geral de Pediatria do Hospital das Clínicas da Universidade Estadual de Campinas (Unicamp). Foi estimada uma média de 400 atendimentos mensais, em um universo de 1 ano de consultas, escolhido aleatoriamente.

O projeto foi desenvolvido no consultório acima referido, porque o mesmo concentra o maior número de consultas ambulatoriais do Departamento de Pediatria (Morcillo et al., 1989), sendo a porta de entrada para as diversas especialidades na área.

A partir da suposição empírica, pela experiência dos profissionais de saúde da área, de uma freqüência de agressão de $10 \%$, foi feito um método de amostragem aleatória sistemática simples, escolhida a partir da primeira criança, com estabelecimento de um intervalo de três crianças, na ordem de chegada à consulta. Foi também considerado o nível de rejeição da hipótese da proporção da referência, um estimador situado no intervalo de confiança de $95 \%$ ou um erro alfa de 0,05 .
Dados esses referenciais, o cálculo amostral foi determinado para a definição de uma proporção em uma população de referência, conforme Fleiss (1981).

A faixa etária pesquisada foi de 0 a 13 anos completos.

A pesquisa foi realizada através de entrevista com o acompanhante da criança, mediante consentimento informado, utilizando-se um protocolo com perguntas abertas, de múltipla escolha ou de escolha única para resposta.

O levantamento de dados ocorreu nos meses de agosto e setembro de 1990, tendo sido o mesmo realizado por quatro pesquisadores.

A teoria sócio-psicológica, ou ecológica, foi utilizada como marco referencial para a elaboração do protocolo, sendo a mesma empregada para a análise do material coletado. Tal teoria considera que os maus-tratos não são resultantes de um único fator, mas de múltiplos fatores que, em interação, produzem circunstâncias oportunas para o surgimento de pais agressores. Assim, haveria uma "ecologia" dos maus-tratos infantis - uma etiologia decorrente da conjugação de características tais como: psicopatologia dos pais ou experiência de maus-tratos na infância, temperamento ou desvio de comportamento, relação conjugal conflituosa ou violenta, privação econômica (Terr, 1991; Vondra \& Toth, 1989; Wolfe et al., 1988).

\section{RESULTADOS}

Os resultados apresentados a seguir referemse aos 130 casos da amostra.

A análise destes casos foi feita através de tabulação simples em tabelas de dupla entrada, analisadas por medidas de qui-quadrado, valor das probabilidades exatas de Fisher e cálculo de odds ratio (Fleiss, 1981).

Observou-se que $89,8 \%$ dos pacientes da amostra procedem do estado de São Paulo, enquanto o restante é proveniente dos estados de Minas Gerais e Paraná.

Em relação à faixa etária, encontrou-se uma distribuição quase uniforme. Em relação ao sexo da criança, $54,6 \%$ eram do sexo masculino e $45,4 \%$, do sexo feminino, sendo que $71,5 \%$ eram brancos, $26,9 \%$ pardos e $1,5 \%$ negros.

Em relação aos entrevistados, $88,5 \%$ eram 
mães das crianças. Destas, 80,8\% eram casadas, com idades variando entre 18 e 70 anos, havendo uma predominância da faixa etária dos 27 aos 34 anos. Além disso, 80,5\% não concluíram o primeiro grau.

Na composição familiar constatou-se a presença simultânea, na maioria dos casos, das figuras paterna e materna, ou substitutivos de tais papéis sociais (114 casos).

Em 42,3\% dos casos, o pai foi o único responsável pela renda familiar, enquanto em $57,7 \%$ o pai e outros membros da família contribuíram para o orçamento doméstico.

Em relação à vida da criança, observou-se que $71,5 \%$ das crianças nasceram hígidas e $27,7 \%$, com enfermidades, sendo que $62,3 \%$ já tinham sido internadas para tratamento de saúde. Em 45,4\% dos casos, a criança foi considerada, pelo entrevistado, diferente de outras crianças, sendo que $69,5 \%$ no aspecto comportamento, 18,6\% em relação à saúde, e o restante em outros aspectos. Existe uma associação entre o entrevistado achar a criança diferente e ele querer mudar algo na mesma (Tabela 1). Encontrou-se odds ratio (OR) de 8,55 para o desejo de mudar o comportamento da criança quando esta é considerada diferente. Neste cruzamento, o objetivo era saber se considerar a criança diferente implicaria desejar mudá-la.

TABELA 1. Avaliação da Aparência do Comportamento e da Saúde Geral da Criança Segundo o Desejo do Responsável de Mudar estes Aspectos na Mesma.

\begin{tabular}{lll}
\hline & \multicolumn{2}{c}{ Mudaria } \\
\cline { 2 - 3 } Criança & Sim & Não \\
Diferente & & \\
\hline Sim & 48 & 11 \\
Não & 24 & 47 \\
\hline Total & 72 & 58 \\
\hline \hline
\end{tabular}

Odds ratio $=8,55$

IC de $95 \%(3,52<$ OR $<21,20 ; \mathrm{p}<0,0001)$

Em 84,6\% dos casos, os entrevistados foram criados pelos pais, não havendo nenhum caso em que a criação foi realizada somente pelo pai.
Em 77,7\% dos casos, a relação do entrevistado com a mãe foi considerada boa; em relação ao pai, este percentual foi de $74,0 \%$.

Em 69,2\% dos casos o, entrevistado foi disciplinado com o uso de força física, sendo que $64,1 \%$ aprovam a maneira como foram disciplinados. Ao se considerar a história de vida dos pais ou responsáveis entrevistados, constatou-se a importância do bom relacionamento dos mesmos com seus genitores - os avós da criança objeto da pesquisa - em relação à aprovação da maneira como foram disciplinados quando eram crianças.

Os comportamentos disciplinares são modelados de uma geração de pais para outra (Schimtt \& Kempe, 1975), sendo constatado um odds ratio de 6,4 para as mães, e de 3,9 para os pais, terem boas relações com os avós e aprovarem seus métodos disciplinares.

A relação do entrevistado com o cônjuge foi considerada boa em $80,9 \%$ dos casos. O entrevistado ou o cônjuge já fez tratamento psiquiátrico em 12,3\% dos casos. Na pesquisa dos hábitos, em 25,4\% dos casos foram referidos problemas com o uso de bebidas alcóolicas e em $2,3 \%$, com o uso de drogas.

Constatou-se que $96,9 \%$ dos entrevistados não usam de força física como atitude preferencial no disciplinamento das crianças. Quando esta atitude não resolve, 40,0\% usam de força física. Considerando-se o uso de força física (como primeira ou segunda atitude), 42,3\% dos entrevistados referiram fazer uso de tal medida, e nos casos onde houve uso de força física, $18,4 \%$ das crianças ficaram com marca roxa ou sinal de agressão (lesão corporal).

A prevalência encontrada na amostra foi de $10,8 \%$ de crianças agredidas, dado que se encontra dentro do intervalo de confiança estimado para o planejamento da pesquisa. Quando a força física é utilizada, 92,7\% escolhem o local do corpo para o emprego da mesma, sendo que $73,5 \%$ escolheram as nádegas, sob a justificativa de que "não machuca" ou "machuca menos".

Encontrou-se, neste trabalho, um risco 2,17 vezes maior para uma criança disciplinada com uso da força física ser considerada diferente pelos seus responsáveis (Tabela 2). A mesma relação não pode ser provada quando analisadas as crianças vítimas de violência, segundo o 
conceito adotado para este trabalho. Embora o risco seja de 2,38 para que uma criança vítima de agressão seja considerada diferente, o intervalo de confiança deixou o risco indefinido $(0,66<$ OR $<8,87 ; \mathrm{p}<0,2)$. Acredita-se que esta dificuldade possa ser atribuída à flutuação amostral (Tabela 3).

TABELA 2. Emprego de Força Física Contra as Crianças como Método Disciplinar em Relação ao Fato de a Mesma ser Considerada Diferente das Demais Crianças.

\begin{tabular}{lcc}
\hline & \multicolumn{2}{c}{ Emprega Força Física } \\
\cline { 2 - 3 } Diferente & Sim & Não \\
\hline Sim & 31 & 28 \\
Não & 24 & 47 \\
\hline Total & 55 & 75 \\
\hline
\end{tabular}

Odds ratio $=2,17$

IC de $95 \%(1,0<$ OR < 4,73); $<<0,048)$

TABELA 3. Emprego de Violência Contra a Criança em Relação ao Fato de a Mesma ser Considerada Diferente das Demais Crianças

\begin{tabular}{lcc}
\hline & \multicolumn{2}{c}{ Emprega Violência } \\
\cline { 2 - 3 } Diferente & Sim & Não \\
\hline Sim & 9 & 50 \\
Não & 5 & 66 \\
\hline Total & 14 & 116 \\
\hline \hline
\end{tabular}

Odds ratio $=2,38$

IC de $95 \%(0,66<$ OR $<8,87) ; \mathrm{p}<0,22)$

\section{DISCUSSÃO}

A prevalência de agressão física contra crianças encontrada neste trabalho foi de $10,8 \%$, compatível com o cálculo amostral estimado. De acordo com a bibliografia internacional, citada por Azevedo \& Guerra (1989), estima-se que $10 \%$ das crianças com menos de 5 anos que se apresentam aos prontos-socorros ou hospitais são vítimas de abuso físico. Lippi (1990) comenta que a estimativa de crianças maltratadas, na faixa etária de 0-14 anos, em 1990, foi de $11,99 \%$.

Neste trabalho, a distribuição por faixa etária não se mostrou significativa, sendo, portanto, compatível com aqueles autores (Hamilton, 1989; Schitt \& Kempe, 1975) que não concordam com a importância da idade na ocorrência de agressões contra a criança.

Segundo Gil (1986), as crianças mais novas correm mais riscos de agressão por serem mais frágeis e suscetíveis a ferimentos, possibilitando o diagnóstico sem muitas dificuldades. Crianças mais velhas são pouco denunciadas, porque o comportamento adolescente é considerado "irreverente", justificando atos abusivos (Gil, 1986). Gelles \& Cornell (1986) colocam que o período de risco para a agressão não deve ser considerado, porque as pesquisas são falseadas, visto serem as mesmas realizadas a partir de casos constatados, e crianças mais velhas são pouco denunciadas porque, sendo menos vulneráveis à lesões físicas, não são registradas pelas pesquisas médicas. Segundo dados da Fibge (1987), as crianças negras e pardas residem em famílias nitidamente mais pobres do que as brancas. Segundo Gil (1971), "uma das causas sociais para o maltrato infantil refere-se à extensão do uso da força física na criação de filhos de famílias de diferentes classes sociais e grupos étnicos". Sobre a importância do aspecto econômico, que poderia justificar o maior índice de agressão em crianças negras, Giovannoni (1974) coloca que as famílias que maltratam os filhos são, elas próprias, vítimas do estresse da pobreza e têm sido privadas do auxílio da comunidade, que logicamente colaboraria para o seu desempenho enquanto pais. Os dados obtidos em relação às variáveis acima mencionadas (renda, idade e cor) não foram compatíveis com os da literatura, pois não foram encontradas relações estatisticamente significativas entre estas variáveis e a variável agressão.

A criança foi considerada diferente das demais em algum aspecto, ocorrendo também maior desejo de mudança da mesma nos casos onde foi constatado o uso de força física abusiva. Estes dados coincidiram com os da literatura na ênfase em relação à contribuição dos 
valores centrados na criança: sexo, prematuridade, alterações congênitas e temperamento contribuem para a ocorrência de violência física contra a criança, ainda que não ocorram isoladamente, mas na interação com os pais, estabelecendo uma relação bidirecional (Gelles, 1973; Gil, 1975; Hamilton, 1989).

Em relação à atitude preferencial utilizada pelos entrevistados para disciplinar, constatouse a predominância da escolha pelo não-uso de força física; recorrendo-se ao uso da mesma quando aquela atitude mostrar-se ineficiente.

Os dados encontrados atestam a hipótese de que, no sentido de disciplinar e educar, a força física é utilizada quando outros métodos disciplinares (não-físicos) mostram-se ineficientes, como conversar ou chamar a atenção. Segundo Gelles \& Cornell (1986), o principal objetivo de se bater em uma criança é ensinar-lhe uma lição, fazê-la mudar um certo comportamento. A violência doméstica, ainda que possa ocorrer com fins pretensamente educativos, na realidade supera o entendimento da violência sobre este prisma: "a pesquisa tem demonstrado repetidas vezes que os maus-tratos não são resultados de um único fator, como a psicopatologia dos pais, o temperamento das crianças, privação econômica, problema no trabalho ou modelos sócio-culturais que encorajam os pais à punição e ao autoritarismo. Cada um destes fatores interagem e juntam-se para produzir circunstância que são oportunas para o surgimento de pais agressivos ou negligentes" (Vondra \& Toth, 1989).

\section{CONCLUSÃO}

O exposto acima leva-nos a concluir que, ainda que possamos pesquisar a ocorrência do uso de força física a partir de uma justificativa de disciplinamento, a violência ultrapassa em muito este âmbito e, devido à conjunção de vários fatores, mostra-se de difícil detecção em protocolo com questões quantitativas.

\section{RESUMO}

DAVOLI, A.; PALHARES, F. A. B.; CORRÊA-FILHO, H. R.; Dias, A. L. V.; ANTUNES, A. B.; SERPA, J. F. \& SCHINCARIOL, P. Prevalência de Violência Física Relatada contra Crianças em uma População de Ambulatório Pediátrico. Cad. Saúde Públ., Rio de Janeiro, 10 (1): 92-98, jan/mar, 1994.

Foi realizado um estudo transversal para se estimar a prevalência da violência física contra crianças de 0 a 13 anos, enquanto método disciplinar, em pacientes do Ambulatório de Pediatria do Hospital de Clínicas da Universidade Estadual de Campinas. A cidade conta com 1 milhão de habitantes com cobertura universal de serviços de saúde. Foi aplicado um questionário a 130 responsáveis que trouxeram as crianças à consulta ambulatorial, através de um esquema aleatório de seleção. A prevalência de crianças agredidas foi de $10,8 \%$. O critério adotado considerou como agredidas as crianças cujos responsáveis relataram o uso de força física, batendo em menores de 1 ano, ou crianças com mais de 1 ano que apresentaram lesões após a agressão física. A aplicação do questionário evidenciou a necessidade de se realizar a avaliação do mesmo enquanto instrumento de medida quantitativa em uma população, visando futuros estudos de prevalência ou incidência. Evidenciou-se também a necessidade do emprego de métodos quantitativos para se aprofundar a pesquisa sobre a gênese sócio-psicológica da agressão física com finalidade supostamente disciplinatórias.

Palavras-Chave: Prevalência; Criança; Maus-Tratos Infantis; Comportamento Materno; Relações Pais-Filhos

\section{REFERÊNCIAS BIBLIOGRÁFICAS}

AZEVEDO, M. A. \& GUERRA, V. N. A. (Orgs.), 1989. Crianças Vitimizadas: A Síndrome do Pequeno Poder. São Paulo: Iglu.

BAEZA-HERRERA, C.; ROQUE, S.; JAMES, S. M. \& FRANCO-VAQUÉZ, R., 1986. Síndrome del niño maltratado - Espectro de un problema, México, 1986. Boletin Medico del Hopital Infantil de Mexico, 43: 71-77. 
CHARLOT, B., 1986. A Mistificação Pedagógica Realidades Sociais e Processos Ideológicos na Teoria da Educação. $2^{\mathrm{a}}$ ed., Rio de Janeiro: Guanabara.

FIBGE (Fundação Instituto Brasileiro de Geografia e Estatística), 1987. Crianças e Adolescentes Indicadores Sociais. Vol. 1, Rio de Janeiro: Fibge.

FLEISS, J. L., 1981. Statistical Methods for Rates and Proportions. $2^{\text {nd }}$ edition, New York: John Wiley \& Sons.

GARBARINO, J. \& GILLIAM, G., 1981. Understanding Abusive Families. Massachusetts: Lexington Books.

GELLES, R. J., 1973. Child abuse as psychopathology: A sociological critique and reformulation. American fournal of Orthopshychiatry, 43: 611-621.

GELLES, R. J. \& CORNELL, C. P., 1986. Intimate Violence in Families. London: SAGE Publications.

GIL, D. G., 1971. A sociocultural perspective on physical child abuse. Child Welfare, 50: 389395.

, 1975. Unravelling child abuse. American Fournal of Ortopsychiatry, 45: 346-356.

, 1986. Violence Against Children Physical Abuse in the United States. $8^{\mathrm{a}}$ ed., Cambridge: Harvard University Press.

GIOVANNONI, J. M., 1974. Parental mistreatment: Perpetrators and victms. Fournal of Marriage and the Family, 33: 649-657.

HAMILTON, L. R., 1989. Variables associated with child maltreatment and implications for prevention and treatment. Early Child Development and Care, 42: 31-56.
KEMPE, C. H.; SILVERMAN, F. N. \& STEELE, B. F., 1962. The battered child syndrome. Fournal of American Medical Association, 181: 17-24.

LIPPI, J. R. S., 1990. Abuso e Negligência na Infância, Prevenção e Direitos. Rio de Janeiro: Editora Científica Nacional.

MORCILLO, A. M.; MARMO, D. B.; BERNARDI, L. \& SIGUEIRA, S. M., 1989. Caracterização da população atendida e da capacidade resolutiva do Ambulatório Geral de Pediatria do Hospital das Clínicas da Unicamp. Revista da Faculdade de Ciências Médicas da Unicamp, 1: 25-32.

SCHIMITT, B. D. \& KEMPE, C. H., 1975. Neglect and abuse of children. In: Textbook of Pediatrics (V. D. Vaughan; R. J. McKay \& W. E. Nelson, eds.), $10^{a}$ ed., pp. 107-111, Philadelphia, Pensylvania: Saunders.

TERR, L., 1981. Childhood trauma: An outline and overview. American Fournal of Psychiatry, 148: 10-20.

TULHA, O. M. P. A., 1979. O vínculo mãe-filho e as necessidades emocionais da criança. In: $A c ̧ \tilde{a} o$ Profilática da Pediatria (D. V. M. Machado, coord.), pp. 08-20, São Paulo: Savier.

VONDRA, J. I. \& TOTH, S., 1989. Ecological perspectives on child maltreatment: Research and Intervention. Early Child Development and Care, 42: 11-29.

WOLFE, D.; EDWARD, D.; MANION, I \& KOVEROLA, C., 1988. Early intervention for parents at risk of child abuse and neglect: A preliminary investigation. Fournal of Consulting and Clinical Physchology, 5: 40-47. 